\title{
Long-Term Risk of Mortality Associated with Isolation of Pseudomonas aeruginosa in COPD: A Systematic Review and Meta-Analysis
}

\author{
Miguel Angel Martinez-García (Did ${ }^{1,2}$, David Rigau ${ }^{3}$, Miriam Barrecheguren ${ }^{2,4}$, Alberto García-Ortega', \\ Alexa Nuñez $\mathbb{D}^{2,4}$, Grace Oscullo Yepez $\mathbb{D}^{1}$, Marc Miravitlles $\mathbb{( D}^{2,4}$ \\ 'Pneumology Department, Hospital Universitario y Politécnico La Fe, Valencia, Spain; ${ }^{2}$ CIBER de Enfermedades Respiratorias (CIBERES), Instituto de \\ Salud Carlos III, Madrid, Spain; ${ }^{3}$ Biomedical Research Institute Sant Pau (IIB Sant Pau), Barcelona, Spain; ${ }^{4}$ Pneumology Department, Hospital \\ Universitari Vall d'Hebron, Vall d'Hebron Institut de Recerca (VHIR), Vall d'Hebron Barcelona Hospital Campus, Barcelona, Spain \\ Correspondence: Marc Miravitlles, University Hospital Vall d'Hebron, Department of Pneumology, Vall d'Hebron Barcelona Hospital Campus, Pg Vall \\ d'Hebron I19-129, Barcelona, 08036, Spain, Email marcm@separ.es
}

Background: Chronic bronchial infection is frequent in chronic obstructive pulmonary disease (COPD), but the impact of the isolation of pathogenic bacteria, and in particular Pseudomonas aeruginosa (PA) in respiratory samples on the prognosis of COPD is unclear.

Methods: We conducted a systematic review of prognostic studies including patients with isolation of PA in sputum in stable state or during exacerbations of COPD. The main outcomes were all-cause mortality, respiratory mortality, and number and severity of future exacerbations. Data were expressed as hazard ratio (HR) (95\% confidence interval [CI]) whenever possible.

Results: Of 2773 studies, eight were finally included (23,228 individuals). The mean age ranged from 65.5 to 73 years. Six studies reported data for all-cause mortality. The adjusted risk of death was almost double in patients with PA isolation (HR $1.95,95 \%$ CI, 1.34 to 2.84; quality of evidence moderate). Patients with PA isolation showed a three times higher adjusted risk of readmission at 30 days after discharge (OR 3.60, 95\% CI, 3.60 to 12.03, 1 study; quality of evidence very low), and more than double adjusted risk of death and hospitalization at two years (HR 2.80, 95\% CI, 2.20 to 3.56, 1 study; quality of evidence very low).

Conclusion: There is moderate certainty that the isolation of PA in sputum is associated with an adjusted increased risk of death in patients with COPD.

Keywords: COPD, prognosis, outcomes, mortality, exacerbations, bronchial infection, bronchial colonization

\section{Introduction}

Both acute and chronic bronchial infection are important features in the natural history of chronic obstructive pulmonary disease (COPD). ${ }^{1}$ Potentially pathogenic microorganisms (PPM) can be isolated from respiratory samples in $30-50 \%$ of clinically stable COPD patients. ${ }^{1,2}$ Some studies suggest that the isolation of PPM could be associated with more frequent exacerbations, ${ }^{3}$ poorer quality of life and clinical outcomes, ${ }^{4}$ increased bronchial and systemic inflammation ${ }^{5,6}$ and even incident cardiovascular diseases. ${ }^{7}$ However, COPD guidelines give little attention to this particular "infective" COPD phenotype, probably because no treatments available to date have shown to be effective for improving strong outcomes in these patients. ${ }^{8,9}$

Pseudomonas aeruginosa (PA) is one of the most virulent PPM in terms of clinical outcomes and mortality in some chronic inflammatory airway diseases such as bronchiectasis ${ }^{10,11}$ or cystic fibrosis, ${ }^{12}$ in which the diagnosis and treatment of the infection by PA is the cornerstone of patient management. ${ }^{13,14}$ However, the impact of PA infection on patients with stable COPD is controversial. While some studies have shown an association between single or multiple PA isolations and poor outcomes including increased mortality, ${ }^{15-17}$ other authors have not found this relationship ${ }^{18,19}$ considering that PA isolation is only a marker of COPD severity not a risk factor for a worse prognosis. ${ }^{20}$ 
Given that PA infection could potentially be effectively treated with antibiotics and anti-inflammatory drugs, ${ }^{13,14}$ that COPD is often associated with bronchiectasis (a disease with a demonstrated high prevalence of PA infection), ${ }^{21}$ and that the relationship between PA infection and mortality in COPD remains controversial, the objective of this study was to conduct a systematic review and meta-analysis of the literature to assess the impact of PA isolation in patients with stable COPD on long-term outcomes including exacerbations, hospitalisations and mortality.

\section{Method}

The systematic review methods and meta-analysis procedures were conducted following published guidelines to develop reviews of prognostic factors. ${ }^{22}$ The protocol was registered in PROSPERO (CRD42020212642) in October 2020 and this article follows the PRISMA guidelines for reporting systematic reviews and meta-analyses (http://prisma-statement.org/).

\section{Inclusion Criteria}

This review included studies verifying the following criteria: A) Types of study: we included observational studies (prospective cohort studies, retrospective cohort studies and case-control studies). The studies should be Phase 2 confirmatory studies, corresponding to explanatory research aimed at confirming an independent association between a potential prognostic factor (in our case the presence of PA) and the outcome of interest. However, Phase 1 exploratory studies could also be included, if assessment of the presence of PA was one of the main aims of the study. ${ }^{23}$ Phase 1 prognostic research corresponds to exploratory studies aimed at investigating all associations, usually by univariate analyses, of potential prognostic factors and outcomes.

B) Condition: COPD and PA in the airways, defined by the isolation of PA in respiratory samples. C) Participants: Patients with the diagnosis of COPD according to international consensus (9), including stable and/or exacerbated COPD. D) Exposures: The index prognostic factor was the presence of PA in a respiratory sample. No requirement was set regarding the method of assessment of the prognostic factor and the choice of cut point value for dichotomising culture results into positive or negative. E) Outcomes: The studies had to report data on any outcome for which the prognostic ability of the presence of PA was of interest, assessed when the participant was in stable condition.

\section{Outcomes}

The main outcomes of the study were as follows: 1) all-cause mortality, 2) mortality due to respiratory causes, 3) exacerbations, 4) Hospitalisation due to COPD exacerbation. Secondary outcomes were forced expiratory volume in one second $\left(\mathrm{FEV}_{1}\right)$ and $\mathrm{FEV}_{1}(\%$ predicted) and quality of life.

\section{Timing}

The prognostic factor of interest (presence of PA) could have been measured at any time after the diagnosis of COPD and with the patient in either a stable or exacerbated state. Outcomes should have been assessed after the determination of exposure, but there was no minimum follow-up required for assessment of outcomes.

\section{Setting}

PA determination could have been conducted in any setting (primary, secondary or tertiary care).

\section{Search Strategy}

A bibliographic search was conducted in MEDLINE (via PubMed) and Embase, retrieving all references up to October 2020. The search combined key controlled-language terms (MESH terms) with free language terms. Given the aim of the review to include Phase 2 confirmatory studies on the prognostic role of PA in COPD, as well as the difficulty to identify prognostic studies, ${ }^{22}$ and the need to avoid an unmanageable search which retrieved a large number of irrelevant results (eg, a search that is too sensitive), the bibliographic search was built to be sensitive but precise, identifying prognostic studies in COPD patients which had a clear focus on PA. For this reason, the term PA had to be either a MESH term or appear in the abstract or title. No language restriction was applied. The detailed search strategy and its results are presented in the Table S1. 
A top-up search was conducted in MEDLINE (via PubMed), EMBASE, Scopus and Web of Science on 21st December 2021, which retrieved 603 additional references (345 after removing duplicates), but did not identify any additional study to be included in the review (Figure 1).

\section{Selection of Studies and Data Extraction}

Two reviewers independently assessed the titles and abstracts of all identified citations and coded them as "retrieve" (eligible or potentially eligible/unclear) or "do not retrieve". The same reviewers then independently evaluated the full text of the articles that at least one reviewer deemed potentially eligible; disagreements were resolved by consensus. The selection of studies was conducted with the on-line platform Covidence (www.covidence.org)

One reviewer independently extracted data using standardised data extraction forms specifically built as an Excel spread sheet. Another reviewer double-checked all source data generated.

\section{Assessment of Risk of Bias and Quality of Evidence}

Two reviewers independently assessed the risk of bias of individual studies using the Quality In Prognosis Studies (QUIPS) tool for assessing the risk of bias in studies of prognostic factors. ${ }^{24}$

The QUIPS tool identifies 6 important areas to consider when evaluating validity and bias in studies of prognostic factors: study participation, study attrition, prognostic factor measurement, confounding measurement and account, outcome measurement, and analysis and reporting. Each domain is assessed through a number of signalling questions, and a judgement is made of 'High, "Moderate", or "Low risk of bias for the domain. An overall judgement of "High, "Moderate", or "Low" risk of bias was derived for each study. ${ }^{22}$ The risk of bias by outcome assessment was assessed separately for each of the three main outcomes (exacerbations, hospitalisations and mortality) to identify differences in bias profile depending on the outcome considered (Supplementary Material 1 and Tables S2a and S2b).

We pre-defined a core set of three confounding factors that were deemed necessary for the primary studies to have adjusted for when formulating the judgement related to risk of bias in the confounding measurement domain. These factors were 1) age, 2) severity of COPD based on a FEV 1 measure, and 3) the presence of bronchiectasis. A table with the ratings of risk of bias for each study was designed reporting each domain separately.

Assessment of quality of evidence followed current guidelines issued by the GRADE Working Group. ${ }^{25}$ Like intervention questions, the GRADE approach suggests five domains for rating down certainty in the evidence: risk of bias, imprecision, inconsistency, indirectness, and publication bias, in addition to the domain for rating up. We applied a noncontextualized situation which implies that there is no direct clinical action associated with the prognostic factor evidence.

Summary of findings tables, including qualitative assessment of the certainty in the evidence and a quantitative description of the relative and absolute effects, were built for each primary outcome except for mortality due to respiratory causes, since none of the studies included reported this outcome.

\section{Data Synthesis}

As a measure of effect, the prognostic value of PA on the outcomes considered was measured as hazard ratios (HR) for time-to-event outcomes and odds ratio (OR) or risk ratio (RR) for dichotomous outcomes. When available in the studies included, adjusted measures of effect were preferred to unadjusted measures.

Statistical heterogeneity was assessed through $\mathrm{I}^{2}$. As a high degree of heterogeneity was expected, no threshold was established for $\mathrm{I}^{2}$, and the decision to meta-analyse was taken purely on clinical grounds. If enough data had been available, sources of heterogeneity would have been explored through pre-specified subgroups: a) population, stable or exacerbated $\mathrm{COPD}$; b) severity, $\mathrm{FEV}_{1}(\%)<50 \%$ or $>50 \%$, and c) phenotype, with or without bronchiectasis; and sensitivity analyses, stratifying into adjusted and unadjusted results.

\section{Data Analysis}

We provided a narrative synthesis of the findings from the studies included, structured around the measurement of the exposure, target population characteristics, description of outcome measurement and characteristics, and the prognostic effect of the exposure. 
2773 records identified through

database searching

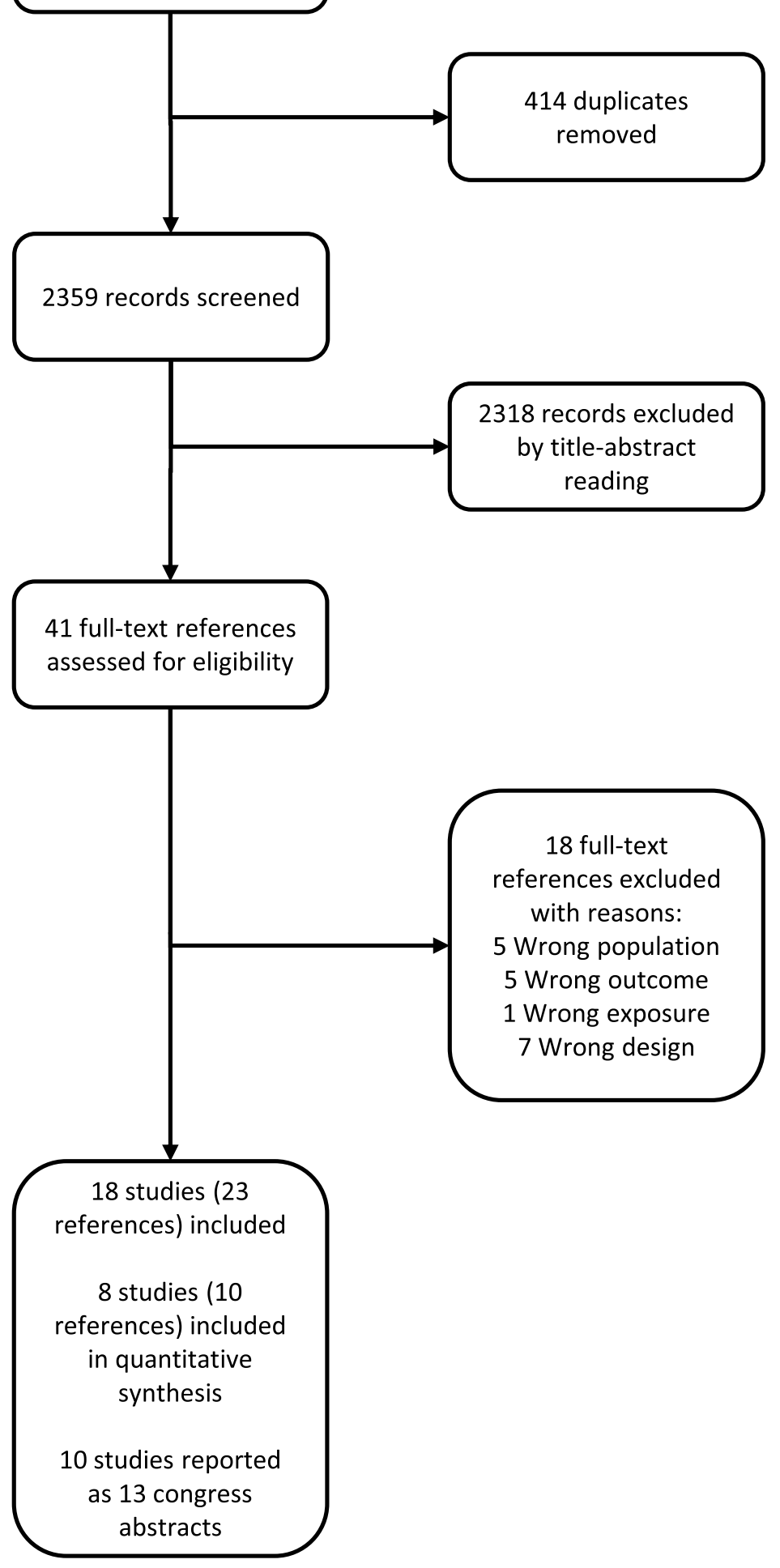

Figure I Flow chart of study selection. 
We organized results by outcome, effect measure used (when outcomes were considered time-to-event or binary) and adjustment or no adjustment of the effect measure, according to guidelines. ${ }^{22}$ However, results have been synthetized across all time points of the outcome occurrence (follow-up duration).

When meta-analysis was deemed appropriate, we pooled comparable results (eg, results for similarly defined outcomes, presented using the same effect measure) through a random-effects meta-analysis using the inverse-variance method, and calculated $95 \%$ confidence intervals $(95 \% \mathrm{CI})$ for the summary effect measure.

Whenever possible we focused on adjusted results, unadjusted results were described as a sensitivity analysis. All statistical analyses were conducted with Review Manager 5.4 software.

\section{Results}

\section{Description of Included Studies}

The search identified 2773 records, and after removing duplicates and verifying inclusion criteria 23 publications from 18 studies were left. Of these, 10 studies were reported in 13 congress abstracts; two of these references corresponded to studies already included. ${ }^{15,19}$ The remaining studies were not included in the systematic review due to the limited information available in the abstract, which prevented assessment of risk of bias and quantitative synthesis of results (Figure 1). Detailed characteristics of the studies included are presented in Table 1 and in Tables S3 and S4.

Finally, this review included 8 studies ${ }^{10,15-19,26,27}$ reporting on a total of 23,228 individuals (range 104 to 22,053) mainly conducted in Europe and USA. The studies included spanned over 20 years, with a follow-up ranging from 36 to 85 months and 6 had a prospective design.

All studies but one ${ }^{18}$ reported a higher percentage of male participants. The mean age ranged from 65.5 to 73 years. Two studies included only severe COPD defined by $\mathrm{FEV}_{1} \%$ levels, ${ }^{26,27} 5$ studies included moderate to severe $\mathrm{COPD}^{10,15-17,19}$ and only one study ${ }^{18}$ also included participants with mild disease (Table 2). Six studies identified PA in sputum cultures ${ }^{10,15-19}$ and 2 also used analysis of bacterial genomic DNA. ${ }^{26,27}$

The percentage of participants with bronchiectasis was reported in four studies and ranged widely from $1.1 \%$ to $62.9 \%$. There was scarce information on the prevalence of comorbidities, with the Charlson index ranging from 2.2 to 4 . Asthma and bronchiectasis as primary diagnoses were explicit exclusion criteria in four studies, ${ }^{10,16,17,19}$ while they were explicitly included in one ${ }^{15}$ and not mentioned in the remaining three studies (Table 2).

Assessment of the isolation of PA was conducted during an exacerbation ${ }^{16,19,26}$ or, alternatively, other studies recruited COPD patients from outpatient clinics or healthcare databases, and PA isolation could either be conducted in a stable or exacerbated state. ${ }^{10,15,17,18,27}$

Table I Characteristics of the Studies Included

\begin{tabular}{|c|c|c|c|c|c|c|}
\hline Authors, Year & Ref. & Country & Recruiting Period & $\mathbf{N}$ included & Design & $\begin{array}{l}\text { Follow-Up } \\
\text { (Months) }\end{array}$ \\
\hline Almagro et al, 2012 & 16 & Spain & June 2003 - September 2004 & 181 & P cohort & 36 \\
\hline Bouquet et al, 2020 & 27 & $\begin{array}{l}\text { Bulgaria, Czech } \\
\text { Republic, USA }\end{array}$ & Fall $201 \mathrm{I}$ - spring 2014 & 200 & P cohort & 36 \\
\hline Boutou et al, 2014 & 18 & UK & $2000-2012$ & 132 & R cohort & NR \\
\hline Choi et al, 2018 & 26 & Korea & January 20II - May 2017 & 104 & $\mathrm{R}$ case-control & 0.5 \\
\hline Eklöf et al, 2020 & 15 & Denmark & January 2010 - October 2017 & 22.053 & P cohort & 36 \\
\hline Jacobs et al, 2020 & 17 & USA & $1994-2014$ & 181 & P cohort & 42.5 \\
\hline Martínez-García et al, 202। & 10 & Spain & January 2004 - February 2007 & 170 & P cohort & 85 \\
\hline Rodrigo-Troyano et al, 2018 & 19 & Spain & March 2012 - March 2015 & 207 & P cohort & 12 \\
\hline
\end{tabular}

Abbreviations: Ref, number of the reference in the reference list; NR, Not reported; P, Prospective; R, Retrospective; USA, United States of America; UK, United Kingdom. 
Table 2 Patient Characteristics

\begin{tabular}{|c|c|c|c|c|c|c|c|c|}
\hline Author, Year (Ref.) & $\begin{array}{l}\text { Male, } \\
\text { n (\%) }\end{array}$ & Age, Years & COPD Severity & FEVI (\%) & $\begin{array}{l}\text { Smokers } \$ \text {, } \\
\text { n (\%) }\end{array}$ & BMI & $\begin{array}{l}\text { Bronchiec- } \\
\text { tasis, n (\%) }\end{array}$ & $\begin{array}{l}\text { Charlson } \\
\text { Index }\end{array}$ \\
\hline Almagro et al, 2012 (16) & $172(95)$ & $72 \pm 9.8$ & $\begin{array}{l}\text { Moderate and severe } \\
\text { (GOLD II-IV) }\end{array}$ & $45.2 \pm 14.4$ & $174(96.1)$ & $27.9 \pm 5$ & $46(52 \%)$ & $2.2 \pm 1.3$ \\
\hline Bouquet et al, 2020 (27) & $130(65)$ & $65.5(50-93)$ & Severe (FEVI<50\%) & $38(13-85)$ & $177(88.5)$ & NR & NR & NR \\
\hline Boutou et al, 2014 (18) & $68(51.5)$ & $68 \pm 8.8$ & NR & $33.6 \pm 14.7$ & NR & $24 \pm 6.3$ & NR & NR \\
\hline Choi et al, 2018 (26) & $78(75)$ & $73.0 \pm 8.4$ & Severe & NR & $79(76)$ & NR & NR & NR \\
\hline Eklöf et al, 2020 (15) & 9868 (44.7) & $69(62-76)$ & NR & $49(36-63)$ & $17.022(77.2)$ & $25(2 \mid-29)$ & $249(1.1)$ & NR \\
\hline Jacobs et al, 2020 (17) & NR & $68(6 I-74)$ & NR & $49.4 \pm 6.04$ & $181(100)$ & NR & $17(9.4)$ & $4(3-5)$ \\
\hline Martínez-García et al, 202I (10) & $155(91.2)$ & $70.1 \pm 9.1$ & $\begin{array}{l}\text { Moderate and severe } \\
\text { (GOLD II-IV) }\end{array}$ & $50.1 \pm 12$ & $170(100)$ & $27.8 \pm 4.8$ & $107(62.9)$ & $2.2 \pm 1.4$ \\
\hline Rodrigo-Troyano et al, 2018 (19) & $|7|(83)$ & $72.2(8.5)$ & Severe (92\% GOLD D) & 35 (IQR 2I) & $78(38)$ & 26 (IQR 5) & NR & 4 (IQR 3) \\
\hline
\end{tabular}

Notes: Values are mean \pm SD or median (Interquartile range (IQR or QI-Q3)), unless otherwise indicated. ${ }^{\$}$ Smokers include active and former smokers. Abbreviations: BMI, Body Mass Index; FEVI, Forced expiratory volume in I second; IQR, interquartile range; GOLD, Global Initiative for Obstructive Lung Disease; NR, Not Reported.

\section{Primary Outcomes. All-Cause Mortality}

Six studies reported results for the all-cause mortality outcome, ${ }^{10,15-19}$ four of them provided adjusted estimates of risk (Table 3). ${ }^{10,15-17}$ Despite the degree of clinical heterogeneity among the six studies, the common definition of the outcome allowed a meta-analysis to be conducted. This meta-analysis showed an adjusted increase in mortality risk associated with PA isolation (HR 1.95, 95\% CI,1.34 to 2.84; $\mathrm{I}^{2} 76 \%$; quality of evidence moderate). A sensitivity analysis conducted with all studies providing estimates of risk reached conclusions similar to those of the main analysis (HR 1.83, $95 \%$ CI, 1.30 to $2.57 ; \mathrm{I}^{2} 72 \%$ ) (Figure 2).

Although Martínez-García et $\mathrm{al}^{10}$ reported a similar adjusted mortality risk over 84 months between patients with a single PA isolation compared to those without PA, there was a higher risk of mortality for patients with persistent isolation of PA versus a single isolation (HR 3.06, 95\% CI, 1.8 to 5.2; $\mathrm{p}=0.001$ ), and there was an association between increasing PA isolations and the risk of mortality (HR 1.2, 95\% CI, 1.1-1.4; $\mathrm{p}<0.001$ ).

\section{Primary Outcomes. Hospitalisation Due to COPD Exacerbation}

Hospitalisations were measured differently in the studies included. Two studies assessed the time until hospitalisation due to COPD exacerbations measured as a time-to-event outcome, ${ }^{15,19}$ but their results could not be pooled due to the clinical heterogeneity between them. One study showed a significant risk of hospitalisation associated with PA isolation (HR 2.80, 95\% CI, 2.20 to 3.56 ), ${ }^{15}$ while the other showed no significant risk (HR 0.95, 95\% CI, 0.53 to 1.70 ) (Table 4 , Figure 3). The quality of evidence for this outcome is very low (Table S5).

Two studies assessed the risk of admission due to exacerbation as a dichotomous outcome, ${ }^{19,26}$ but again their results could not be pooled due to the clinical heterogeneity between them in terms of how they defined the outcome and the statistical methods applied. One study assessed the risk of being readmitted within 30 days after discharge for an exacerbation of COPD. In a case-control study Choi et $\mathrm{al}^{26}$ reported an adjusted higher risk of a 30-day readmission rate in PA positive patients (23.1\% versus $7.7 \%$; adjusted OR, 4.74, 95\% CI, 1.29 to 17.40). The second study reported similar unadjusted number of patients with one readmission in PA versus no-PA groups, but a higher percentage of patients with $\geq 3$ readmissions in PA-positive patients at one year follow-up in a cohort of frequently exacerbating patients $^{19}$ (Table 4, Figure 3). The quality of evidence for this outcome is very low (Table S4).

Finally, four studies reported the number of readmissions during follow-up. ${ }^{10,16,17,19}$ They presented clinical heterogeneity in the populations considered, the definition of the type of readmissions, the follow-up period, and the way in which the data were presented. Their results were inconsistent, with two studies reporting a similar number of readmissions in cohorts of patients discharged following an exacerbation of COPD with or without PA isolation, ${ }^{16,19}$ and two studies reported higher numbers of readmissions in PA-positive outpatients ${ }^{10,17}$ (Table 4). The results presented in Figure 3 correspond to the two studies that provided enough data to estimate a measure of 
Table 3 Summary of Results of Mortality in Patients with and without PA Isolation

\begin{tabular}{|c|c|c|}
\hline \multicolumn{3}{|l|}{ Mortality } \\
\hline Study & Data & Follow Up \\
\hline Almagro et al $2012(16)$ & Adjusted mortality risk (HR 2.2, 95\% Cl: I.2-4.2; $\mathrm{p}=0.02)$ & 3 years \\
\hline Eklöf et al 2020 (15) & $\begin{array}{l}\text { Adjusted mortality risk (HR 2.7, } 95 \% \mathrm{Cl}: 2.3-3.4, \mathrm{p}<0.00 \mathrm{I}) \text {. } \\
\text { Sensitivity analysis in bronchiectasis patients: } \mathrm{HR} 2.9(95 \% \mathrm{Cl}: 2.4-3.5)\end{array}$ & 2 years \\
\hline Jacobs et al $2020(17)$ & $\begin{array}{l}\text { Adjusted mortality risk (HR I.78, } 95 \% \mathrm{Cl} \text { : I.24-2.55, } \mathrm{p}=0.00 \mathrm{I}) \\
\text { Sensitivity analysis adjusting for bronchiectasis: HR I.89 }(95 \% \mathrm{Cl}: \text { I.22-2.94; } \mathrm{p}=0.005)\end{array}$ & 3 years \\
\hline Martínez-García et al 202I (I0) & $\begin{array}{l}\text { Adjusted mortality risk of a single PA isolation during follow-up vs no PA (HR I.25, } 95 \% \mathrm{Cl} \text { : } \\
0.79-1.97, \mathrm{p}=0.34 \text { ) } \\
\text { Adjusted risk of mortality of persistent isolation of PA during follow-up vs single isolation } \\
\text { event (HR } 3.06,95 \% \mathrm{Cl} \text { : I.8-5.2, } \mathrm{P}=0.00 \mathrm{I}) \\
\text { Adjusted risk of mortality for the number of PA isolates (HR I.2, } 95 \% \mathrm{Cl} \text { : I.I-I.4, } \mathrm{p}<0.00 \mathrm{I} \text { ) }\end{array}$ & 84 months \\
\hline Boutou et al 2014 (18) & Unadjusted mortality risk (HR I.2I, $95 \% \mathrm{Cl}: 0.70-2.10$, log rank test $\mathrm{p}=0.49$ ). & 81.2 months \\
\hline Rodrigo-Troyano et al 2018 (19) & Unadjusted mortality risk (HR 2.29, 95\% Cl: $0.74-7.12, p=0.15$ ) & I year \\
\hline
\end{tabular}

Abbreviations: HR, Hazard ratio; Cl, Confidence interval; PA, Pseudomonas aeruginosa.

effect. No studies reported results of respiratory mortality. The quality of evidence for this outcome is very low (Table S5).

\section{Primary Outcomes. Exacerbations}

Four studies reported results on exacerbations, but they could not be pooled due to the clinical heterogeneity across them. One study reported a higher adjusted risk of presenting at least one exacerbation event and the risk of presenting two or more exacerbations a year in severe COPD patients with $\mathrm{PA}^{27}$ (Table 4).

Additionally, two studies reported adjusted higher exacerbation rates in cohorts of outpatients who were PA positive, ${ }^{10,17}$ and one study reported a similar number of annual exacerbations in a cohort of frequent exacerbators. ${ }^{19}$ The quality of evidence for this outcome is very low (Table S5).

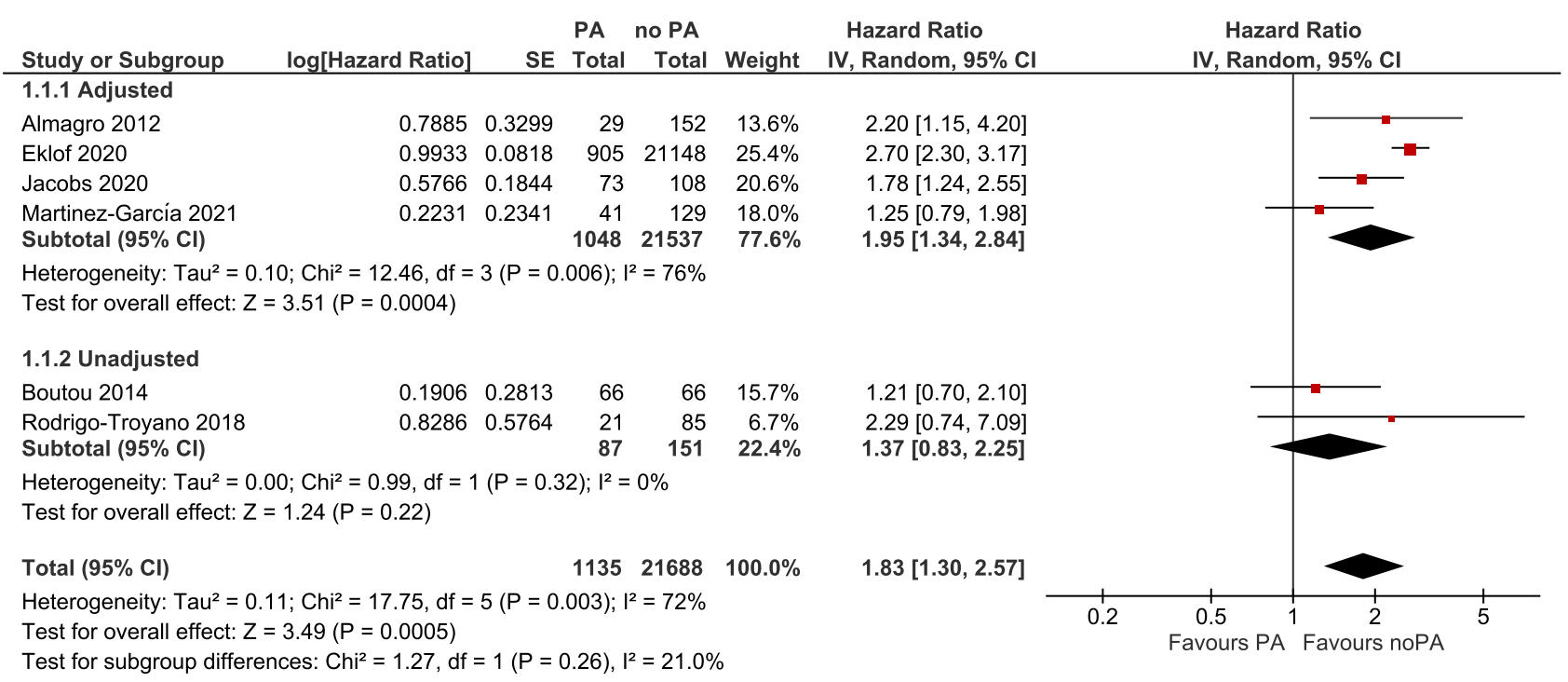

Figure 2 Forest plot of meta-analysis for death. 
Table 4 Summary of Results of Hospitalisations and Exacerbations in Patients with and without PA Isolation

\begin{tabular}{|c|c|c|}
\hline \multicolumn{3}{|l|}{ Time to Hospitalisation } \\
\hline Study & Data & Follow Up \\
\hline Eklöf et al 2020 (15) & $\begin{array}{l}\text { Adjusted risk of the composite outcome hospitalisation for COPD or all-cause death (HR } \\
2.8,2.2-3.6, \mathrm{P}<0.00 \mathrm{I}) \text {. } \\
\text { Sensitivity analysis on bronchiectasis patients: HR } 2.5(95 \% \mathrm{Cl}: 1.9-3.3)\end{array}$ & 2 years \\
\hline Rodrigo-Troyano et al 2018 (19) & Unadjusted Risk of readmission (HR $0.95,0.53-1.68, \mathrm{p}=0.85)$ & I year \\
\hline \multicolumn{3}{|l|}{ Risk of readmission } \\
\hline Choi et al $2018(26)$ & $\begin{array}{l}\text { Propensity score-adjusted readmission risk in patients PA positive vs no PA positive (OR= } \\
3.60,95 \% \mathrm{Cl}: 1.03-8.70,23.1 \% \text { vs } 7.7 \%, \mathrm{p}=0.030) \\
\text { Propensity score and covariables-Adjusted risk (OR=4.75, } 95 \% \mathrm{Cl}: 1.29-17.40)\end{array}$ & 30 days \\
\hline Rodrigo-Troyano et al 2018 (19) & $\begin{array}{l}\text { Unadjusted proportion of patients with one readmission in PA vs no-PA groups ( } 79 \% \text { vs } \\
75 \%, p=0.9 \text { ) } \\
\text { Unadjusted number of patients with }>3 \text { readmissions ( } 33 \% \text { vs II\%, } p=0.01 . \mathrm{OR}=4.1,95 \% \\
\mathrm{Cl}: 1.3-12.8 \text { ) }\end{array}$ & I year \\
\hline \multicolumn{3}{|l|}{ Number of readmissions } \\
\hline Almagro et al $2012(16)$ & Unadjusted mean number of readmissions ( $1.3 \pm 1.5$ vs $1.2 \pm 1.6, p=0.8)$. & I year \\
\hline Rodrigo-Troyano et al 2018 (19) & Unadjusted median number of readmissions/ 1000 follow-up days ( 2.7 vs $2.7, p=0.7$ ) & I year \\
\hline Jacobs et al $2020(17)$ & $\begin{array}{l}\text { Unadjusted COPD-related hospitalisation rates per } 100 \text { persons-month (rate } 1.87 \text { ( } 95 \% \mathrm{Cl} \\
\mathrm{I} .22-2.74) \text { vs } 0.89(95 \% \mathrm{Cl} 0.5 \mathrm{I}-\mathrm{I} .44), \mathrm{p}=0.02) \text {. Unadjusted Rate ratio I. } 15,95 \% \mathrm{Cl} \text { : } 1.59- \\
2.78\end{array}$ & 3 years \\
\hline Martínez-García et al 202 I (10) & Unadjusted mean number of severe exacerbations $(0.76 \pm 1.0$ vs $0.23 \pm 0.4, p=0.003)$ & 84 months \\
\hline \multicolumn{3}{|l|}{ COPD exacerbations } \\
\hline Bouquet et al $2020(27)$ & $\begin{array}{l}\text { Adjusted risk of an exacerbation event (OR I.20, } 95 \% \mathrm{Cl} \text { : I.03-I.39) } \\
\text { Adjusted risk of frequent exacerbations ( } \geq 2 / y)(\mathrm{OR} \text { I.48, } 95 \% \mathrm{Cl}: \text { I.3I-I.68) }\end{array}$ & 3 years \\
\hline Jacobs et al 2020 (I7) & Unadjusted exacerbation rates per 100 person-month $(15.7$ vs $7.5, p<0.00 I)$ & 3 years \\
\hline Martínez-García et al 2021 (10) & $\begin{array}{l}\text { Unadjusted annual total exacerbation rates }(1.87 \pm 1.3 \text { vs } 0.84 \pm 0.8, p=0.009) \text { annual } \\
\text { moderate exacerbation rates }(1.11 \pm 1.3 \text { vs } 0.61 \pm 0.9, p=0.01)\end{array}$ & 84 months \\
\hline Rodrigo-Troyano et al 2018 (19) & Unadjusted number of non-hospitalised exacerbations ( $\mathrm{I}$ vs $\mathrm{I}, \mathrm{p}=0.8$ ) & I year \\
\hline
\end{tabular}

Abbreviations: OR, Odds ratio; HR, Hazard ratio; $\mathrm{Cl}$, Confidence interval; PA, Pseudomonas aeruginosa.

\section{Secondary Outcomes}

One study assessed $\mathrm{FEV}_{1}$ one month after hospital discharge during stable state ${ }^{16}$ and found significant differences between PA-positive and PA-negative patients (unadjusted $\mathrm{FEV}_{1}$ median 907 vs 1081 respectively; $\mathrm{p}=0.02$ and unadjusted $\mathrm{FEV}_{1} \%$ predicted median $38.7 \%$ vs $45.9 \%$ respectively; $\mathrm{p}=0.009$ ). No studies reported on other pre-specified secondary outcomes such as quality of life.

\section{Risk of Bias and Quality of Evidence of the Studies Included}

Three of the studies included presented an overall moderate risk of bias, ${ }^{10,15,17}$ while the remaining studies presented an overall high risk of bias. ${ }^{16,18,19,26,27}$ By domains, we observed moderate to high risk of bias for all domains except that of prognostic factor measurement, which had a low risk of bias. A detailed description of the risk of bias assessment is presented in Table S3. Assessment of the quality of evidence is presented in detail in Table S5. 


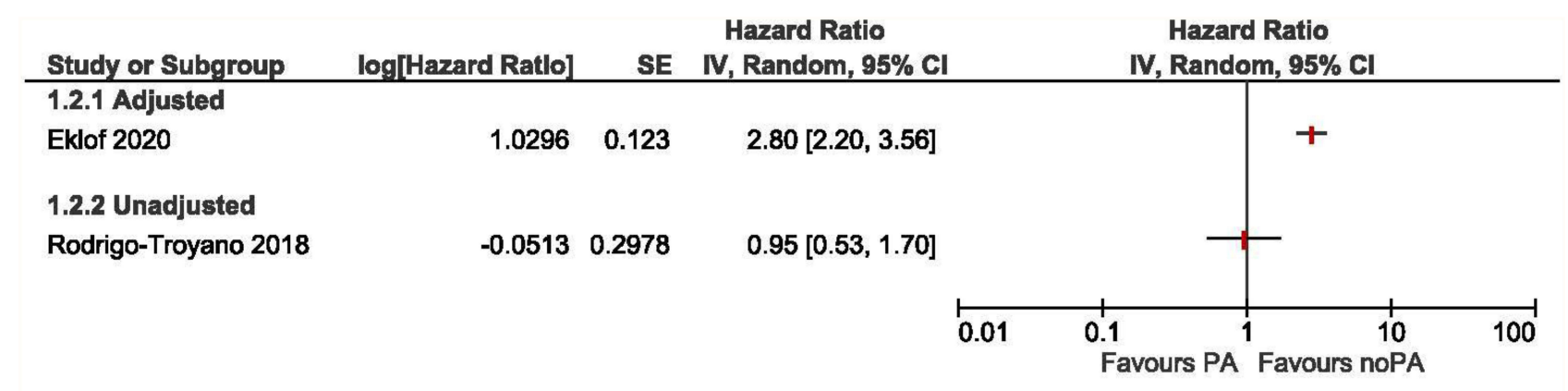

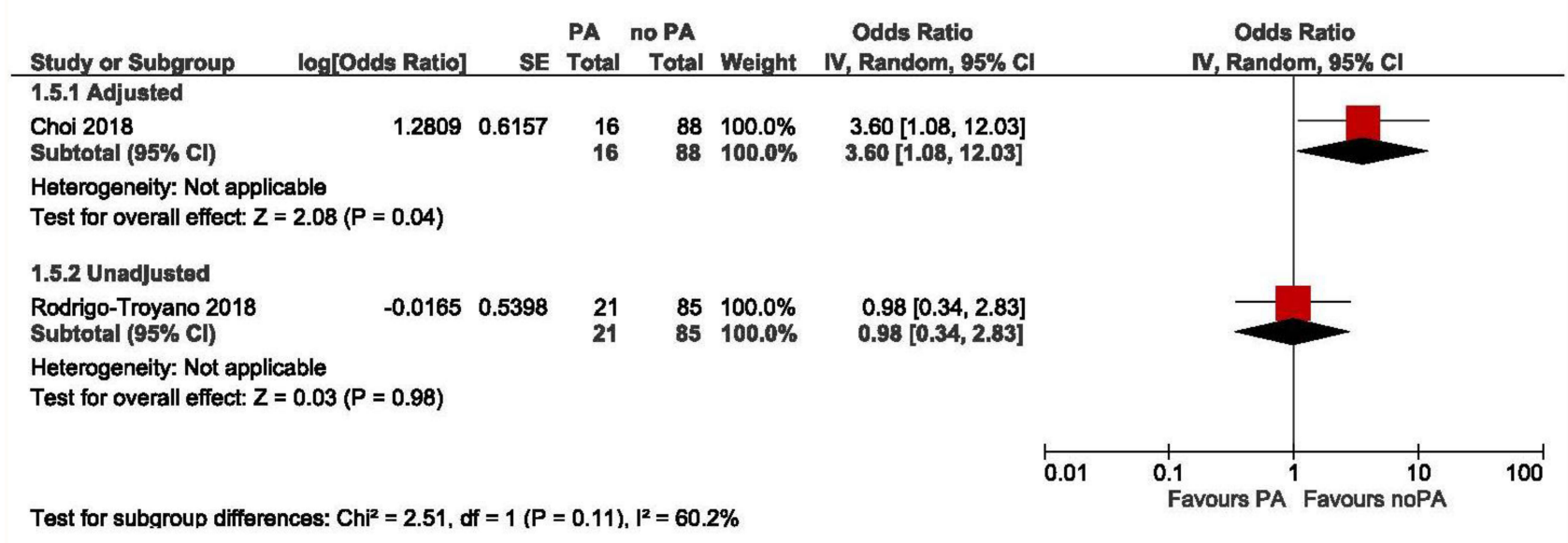

\begin{tabular}{|c|c|c|c|c|c|c|c|c|c|}
\hline \multirow{3}{*}{$\begin{array}{l}\text { Study or Subgroup } \\
\text { Almagro } 2012\end{array}$} & \multicolumn{3}{|c|}{ PA } & \multicolumn{3}{|c|}{ noPA } & & $\begin{array}{l}\text { Mean Difference } \\
\text { IV, Random, 95\% Cl }\end{array}$ & $\begin{array}{l}\text { Mean Difference } \\
\text { IV, Random, 95\% Cl }\end{array}$ \\
\hline & 13 & & 20 & & & 150 & & & \\
\hline & 0.06 & 1.0 & 29 & 1.2 & 1.0 & 102 & & $0.10[-0.00,0.10]$ & \\
\hline & & & & & & & & & $\begin{array}{llll}-1 & -0.5 & 0 & 0.5\end{array}$ \\
\hline
\end{tabular}

Figure 3 Forest plot of meta-analysis for time to hospitalisation, risk of readmission and number of readmissions.

\section{Discussion}

The results of our meta-analysis suggest that the isolation of PA in respiratory samples in patients with COPD, either in stable state or during an exacerbation, is associated with almost double the adjusted risk of all-cause mortality over a follow-up of up to 84 months. Due to the limited amount of data available, the relationship between the isolation of PA in COPD and the risk of exacerbations, hospitalisations, lung function and quality of life was inconclusive.

Single or repeated isolation of PPMs in patients with COPD is not infrequent, even during the stable phase of the disease. In fact, PPMs may be recovered from respiratory samples in 30-50\% of COPD patients over the course of their natural history ${ }^{1,2}$ and they are associated with poorer clinical and functional outcomes. ${ }^{3-7}$ Despite studies showing that PA more frequently infects patients with increasingly severe COPD, ${ }^{20,28}$ evidence of the impact of PA on the prognosis of COPD is scarce.

PA is one of the most virulent PPM, especially in the form of chronic bronchial infection (CBI) and is a key prognostic factor in bronchiectasis, ${ }^{13,14}$ in which CBI by PA is associated with double the adjusted risk of death (HR 2.02, 95\% CI, 1.53 to 2.66), ${ }^{11,29}$ as well as with an accelerated decline in lung function (more than $50 \mathrm{~mL} /$ year of $\mathrm{FEV}_{1}$ ). ${ }^{10}$ However, the impact of PA isolation on COPD prognosis and outcomes remains uncertain. The main results of the current meta-analysis related to mortality are consistent with those observed in patients with bronchiectasis, since PA isolation was associated with almost 
double the risk of death in stable COPD patients. It is important to highlight that this significant increase in the risk of mortality was confirmed in an analysis adjusted for the main confounders, including the severity of COPD, and was observed in five out of the six studies identified. ${ }^{10,15-17,19}$ Moreover, our study did not focus on the acute effect on mortality of the isolation of PA during an exacerbation, but conversely, it investigated the impact of PA isolation on long-term mortality, thereby, assessing the relationship between PA and the natural history of COPD. The only study that did not find a significant increase in mortality risk analysed survival associated with a single isolation of PA in sputum of a group of 66 PA-positive COPD patients compared to 66 PA-negative COPD matched controls. ${ }^{18}$ Their results were like those obtained by Martínez-García et al ${ }^{10}$ in a cohort of 170 COPD patients, in whom no significantly increased mortality was associated with a single isolation of PA, but they did observe a 3-fold increased risk of mortality associated with persistent isolation of PA compared with a single isolation, and a $20 \%$ increase in the risk of death associated with every increase in one positive PA isolation during follow-up. These results suggest that it is not the isolation of PA, but rather its persistence, ie, CBI by PA, which is associated with poor outcomes in COPD and provides the basis for the investigation of CBI in every patient in whom PA is isolated. ${ }^{2,30}$ On the other hand, these data suggest that effective eradication of PA in patients with CBI may potentially be associated with increased survival ${ }^{31,32}$ although future prospective studies are required to confirm this hypothesis.

The association of PA isolation and the risk of exacerbation, hospitalisation and readmission was inconclusive due to the differences in design and data analysis of the studies included. The majority of studies suggested an association between PA isolation and increased risk of exacerbations, but some did not. The largest study carried out in $+22,000$ patients found an adjusted risk of the composite outcome of hospitalisation for COPD or all-cause mortality of HR: $2.8{ }^{15}$ Interestingly, the studies that failed to show a significant association between PA isolation and exacerbation were those including patients during hospital admission and with a very small sample size. Rodrigo-Troyano et al ${ }^{19}$ did not find a significant association between PA and risk of exacerbation, readmission or the proportion of patients with readmission in a cohort of 207 hospitalised patients, of whom only $21(10 \%)$ had a positive culture for PA. Similarly, Almagro et $\mathrm{al}^{16}$ did not find a significantly increased risk of readmission in a group of only 29 (16\%) PA-positive patients from a cohort of 181 patients hospitalised for a COPD exacerbation. It is quite likely that these studies were not powered to detect a difference in exacerbations or readmission rates between patients with and without PA over a period of only one year.

Our study has some limitations. The most relevant is the small number of studies and their heterogeneity, which did not allow to obtain definitive conclusions about the risk of exacerbations or hospitalisations. However, data on the most important outcome, mortality, were less heterogeneous and allowed a meta-analysis with clear results. To the best of our knowledge, this is the first meta-analysis reporting an increased risk of mortality in patients with COPD associated with the isolation of PA, especially in cases of repeated isolation of this microorganism.

The results of this study have important clinical implications. On one hand, it highlights once again the important role of bacterial infection in COPD even in stable state, outside of periods of exacerbation and, therefore, suggests the need for microbiological monitoring of sputum particularly in severe COPD patients with frequent exacerbations. ${ }^{2,33}$ There is limited evidence so far about the efficacy of antimicrobial therapy in CBI, outside of periods of exacerbation, ${ }^{32,34}$ but antimicrobial therapy is indicated if PA is isolated in the context of bronchiectasis. ${ }^{13,14}$

There are still several uncertainties about the role of PPM, and in particular PA in stable COPD. Information about the impact of PA on the exacerbation rate, quality of life, lung function decline and response to treatment, ie, inhaled corticosteroids (ICS), ${ }^{35,36}$ is very scarce and well-designed future prospective studies are urgently needed. In relation to treatment, a study on patients with bronchiectasis from the US Medicare database compared the outcomes between $+83,000$ new users of ICS ( $84.4 \%$ with COPD) and 6500 new users of macrolides (77.7\% with COPD) and found that the use of ICS was associated with an increased risk of hospitalised respiratory infections compared with macrolide monotherapy in the whole group and in the subgroup of patients with COPD. ${ }^{35}$ Interestingly, a very recent study in Denmark showed that ICS use in patients with COPD was associated with a substantially increased and dose-dependent risk of acquiring PA. ${ }^{37}$ Regarding the impact of PPMs in COPD, the limited existing evidence suggest that we should not refer to the isolation of PPM in stable COPD as colonisation but rather as $\mathrm{CBI}$, since it is associated with increased inflammation and worse outcomes. ${ }^{1,2,30,33}$ Finally, an "infective" COPD phenotype should be considered since these patients have unique characteristics and probably require a different therapeutic approach. ${ }^{1}$ Until more evidence about strategies of treatment for these patients is available, we should consider and investigate the presence of a possible overlap between COPD and bronchiectasis ${ }^{38,39}$ and treat both components according to their respective guidelines. 


\section{Conclusions}

The isolation of potentially pathogenic microorganisms in COPD patients may have important clinical implications. Pseudomonas aeruginosa is one of the most virulent and its isolation is associated with increased mortality in COPD patients.

\section{Abbreviations}

CBI, chronic bronchial infection; CI, confidence interval; COPD, chronic obstructive pulmonary disease; FEV1, forced expiratory volume in one second; GRADE, grading of recommendations assessment, development and evaluation; HR, hazard ratio; HI, Haemophilus influenzae; ICS, inhaled corticosteroid; MESH, medical subject headings; OR, odds ratio; PA, Pseudomonas aeruginosa; PPM, potentially pathogenic microorganism; PROSPERO, prospective registry of systematic reviews; QUIPS, Quality In Prognosis Studies; RR, risk ratio; USA, United States of America.

\section{Acknowledgments}

The authors want to thank Marta Roqué, Centro Cochrane Iberoamericano, Barcelona, Spain for her collaboration in the methodology of the study. Alexa Nuñez is the recipient of a Rio Hortega contract in the 2019 Strategic Action Health Call from the Instituto de Salud Carlos III for the years 2020-2022.

\section{Funding}

This study has been funded by an unrestricted grant from Boehringer Ingelheim Spain.

\section{Disclosure}

Miguel Angel Martinez-Garcia has received fees from Chiesi, GlaxoSmithKline, Menarini, Rovi, Bial, Zambon, Vitalire, TEVA, Grifols and Novartis, consulting fees from Grifols, Zambon and TEVA, and research grants from TEVA, Zambon and Vitalaire. David Rigau reports grants from Boehringer Ingelheim. Miriam Barrecheguren has received speaker fees from Grifols, Menarini, CSL Behring, GSK, Boehringer Ingelheim and consulting fees from GSK, Novartis and Boehringer Ingelheim. Marc Miravitlles has received speaker fees from AstraZeneca, Atriva Therapeutics, Boehringer Ingelheim, Chiesi, Cipla, GlaxoSmithKline, Menarini, Rovi, Bial, Sandoz, Zambon, CSL Behring, Grifols and Novartis, consulting fees from AstraZeneca, Boehringer Ingelheim, Chiesi, GlaxoSmithKline, Bial, Gebro Pharma, Kamada, CSL Behring, Laboratorios Esteve, Ferrer, Johnson \& Johnson, Mereo Biopharma, Palobiofarma SL, Takeda, Verona Pharma, TEVA, Spin Therapeutics, pH Pharma, ONO Pharma, Novartis, Sanofi and Grifols and research grants from Grifols. The remaining authors have no conflicts of interest to disclosure.

\section{References}

1. Matkovic Z, Miravitlles M. Chronic bronchial infection in COPD Is there an infective phenotype? Respir Med. 2013;107:10-22.

2. de la Rosa Carrillo D, Lopez-Campos JL, Alcazar Navarrete B, et al. Consensus document on the diagnosis and treatment of chronic bronchial infection in chronic obstructive pulmonary disease. Arch Bronconeumol. 2020;56:651-664.

3. Patel IS, Seemungal TA, Wilks M, Lloyd-Owen SJ, Donaldson GC, Wedzicha JA. Relationship between bacterial colonisation and the frequency, character, and severity of COPD exacerbations. Thorax. 2002;57:759-764.

4. Banerjee D, Khair OA, Honeybourne D. Impact of sputum bacteria on airway inflammation and health status in clinical stable COPD. Eur Respir J. 2004;23:685-691.

5. Sethi S, Maloney J, Grove L, Wrona C, Berenson CS. Airway inflammation and bronchial bacterial colonization in chronic obstructive pulmonary disease. Am J Respir Crit Care Med. 2006;173:991-998.

6. Beech AS, Lea S, Kolsum U, et al. Bacteria and sputum inflammatory cell counts; a COPD cohort analysis. Respir Res. 2020;21:289.

7. Fuschillo S, Martucci M, Donner CF, Balzano G. Airway bacterial colonization: the missing link between COPD and cardiovascular events? Respir Med. 2012;106:915-923.

8. Miravitlles M, Calle M, Molina J, et al. Spanish COPD Guidelines (GesEPOC) 2021: updated Pharmacological treatment of stable COPD. Arch Bronconeumol. 2022;58:69-81.

9. Global strategy for the diagnosis, management, and prevention of chronic obstructive pulmonary disease; 2021. Available from: https://goldcopd. org/wp-content/uploads/2020/11/GOLD-REPORT-2021-v1.1-25Nov20_WMV.pdf. Accessed May 16, 2021.

10. Martínez-García MÁ, Faner R, Oscullo G, et al. Risk factors and relation with mortality of a new acquisition and persistence of Pseudomonas aeruginosa in COPD Patients. COPD. 2021;18:333-340.

11. Martinez-Garcia MA, de Gracia J, Vendrell Relat M, et al. Multidimensional approach to non-cystic fibrosis bronchiectasis: the FACED score. Eur Resp J. 2014;43:1357-1367. 
12. Amin R, Lam M, Dupuis A, Ratjen F. The effect of early Pseudomonas aeruginosa treatment on lung function in pediatric cystic fibrosis. Pediatr Pulmonol. 2011;46:554-558.

13. Martinez-Garcia MA, Maiz L, Olveira C, et al. Spanish Guidelines on the treatment of Bronchiectasis in Adults. Arch Bronconeumol. 2018;54:88-98.

14. Polverino E, Goeminne PC, McDonnell MJ, et al. European Respiratory Society guidelines for the management of adult bronchiectasis. Eur Respir J. 2017;50(3):1700629.

15. Eklof J, Sorensen R, Ingebrigtsen TS, et al. Pseudomonas aeruginosa and risk of death and exacerbations in patients with chronic obstructive pulmonary disease: an observational cohort study of 22053 patients. Clin Microbiol Infect. 2020;26:227-234.

16. Almagro P, Salvado M, Garcia-Vidal C, et al. Pseudomonas aeruginosa and mortality after hospital admission for chronic obstructive pulmonary disease. Respiration. 2012;84:36-43.

17. Jacobs DM, Ochs-Balcom HM, Noyes K, et al. Impact of Pseudomonas aeruginosa isolation on mortality and outcomes in an outpatient chronic obstructive pulmonary disease cohort. Open Forum Infect Dis. 2020;7:ofz546.

18. Boutou AK, Raste Y, Reid J, Alshafi K, Polkey MI, Hopkinson NS. Does a single Pseudomonas aeruginosa isolation predict COPD mortality? Eur Respir J. 2014;44(3):794-797.

19. Rodrigo-Troyano A, Melo V, Marcos PJ, et al. Pseudomonas aeruginosa in Chronic Obstructive Pulmonary Disease Patients with Frequent Hospitalized Exacerbations: a Prospective Multicentre Study. Respiration. 2018;96:417-424.

20. Murphy TF. The many faces of Pseudomonas aeruginosa in chronic obstructive pulmonary disease. Clin Infect Dis. 2008;47:1534-1536.

21. Martinez-Garcia MA, Miravitlles M. Bronchiectasis in COPD patients: more than a comorbidity? Int J Chron Obstruct Pulmon Dis. 2017;12:1401-1411.

22. Riley RD, Moons KGM, Snell KIE, et al. A guide to systematic review and meta-analysis of prognostic factor studies. BMJ. 2019;364:k4597.

23. Hayden JA, Côté P, Steenstra IA, Bombardier C, QUIPS-LBP Working Group. Identifying phases of investigation helps planning, appraising, and applying the results of explanatory prognosis studies. J Clin Epidemiol. 2008;61:552-560.

24. Hayden JA, van der Windt DA, Cartwright JL, Côté P, Bombardier C. Assessing bias in studies of prognostic factors. Ann Intern Med. $2013 ; 158: 280-286$.

25. Foroutan F, Guyatt G, Zuk V, et al. GRADE Guidelines 28: use of GRADE for the assessment of evidence about prognostic factors: rating certainty in identification of groups of patients with different absolute risks. $J$ Clin Epidemiol. 2020;121:62-70.

26. Choi J, Oh JY, Lee YS, et al. Pseudomonas aeruginosa infection increases the readmission rate of COPD patients. Int J Chron Obstruct Pulmon Dis. 2018;13:3077-3083.

27. Bouquet J, Tabor DE, Silver JS, et al. Microbial burden and viral exacerbations in a longitudinal multicentre COPD cohort. Respir Res. $2020 ; 21(1): 77$.

28. Miravitlles M, Espinosa C, Fernández-Laso E, Martos JA, Maldonado JA, Gallego M, and Study Group of Bacterial Infection in COPD. Relationship between bacterial flora in sputum and functional impairment in patients with acute exacerbations of COPD. Chest. 1999;116:40-46.

29. Araújo D, Shteinberg M, Aliberti S, et al. The independent contribution of Pseudomonas aeruginosa infection to long-term clinical outcomes in bronchiectasis. Eur Respir J. 2018;51:1701953.

30. López-Campos JL, Miravitlles M, De la Rosa Carrillo D, Cantón R, Soler-Cataluña JJ, Martinez-Garcia MA. Current challenges in chronic bronchial infection in patients with chronic obstructive pulmonary disease. J Clin Med. 2020;9:E1639.

31. Miravitlles M, Anzueto A. Chronic respiratory infection in patients with chronic obstructive pulmonary disease: what is the role of antibiotics? Int J Mol Sci. 2017;18:E1344.

32. De la Rosa Carrillo D, Martínez-García MÁ, Barreiro E, et al. Effectiveness and Safety of Inhaled Antibiotics in Patients With Chronic Obstructive Pulmonary Disease. A Multicentre Observational Study. Arch Bronconeumol. 2022;58:11-21.

33. Sethi S, Murphy TF. Infection in the pathogenesis and course of chronic obstructive pulmonary disease. N Engl J Med. 2008;359:2355-2365.

34. Miravitlles M, Marín A, Monsó E, et al. Efficacy of moxifloxacin in the treatment of bronchial colonization in COPD. Eur Respir J. 2009;34:1066-1071.

35. Henkle E, Curtis JR, Chen L, et al. Comparative risks of chronic inhaled corticosteroids and macrolides for bronchiectasis. Eur Respir J. 2019;54:1801896.

36. Martinez-Garcia MA, Faner R, Oscullo G, et al. Inhaled Steroids, Circulating Eosinophils, Chronic Airway Infection, and Pneumonia Risk in Chronic Obstructive Pulmonary Disease. A Network Analysis. Am J Respir Crit Care Med. 2020;201:1078-1085.

37. Eklöf J, Ingebrigtsen TS, Sørensen R, et al. Use of inhaled corticosteroids and risk of acquiring Pseudomonas aeruginosa in patients with chronic obstructive pulmonary disease. Thorax. 2021:thoraxjnl-2021-217160. doi:10.1136/thoraxjnl-2021-217160

38. Polverino E, Dimakou K, Hurst J, et al. The overlap between bronchiectasis and chronic airways diseases: state of the art and future directions. Eur Respir J. 2018;52:pii: 1800328

39. Padilla-Galo A, Olveira Fuster C. Bronchiectasis in COPD and Asthma. More Than Just a Coincidence. Arch Bronconeumol. 2019;55:181-182.

International Journal of Chronic Obstructive Pulmonary Disease

\section{Publish your work in this journal}

The International Journal of COPD is an international, peer-reviewed journal of therapeutics and pharmacology focusing on concise rapid reporting of clinical studies and reviews in COPD. Special focus is given to the pathophysiological processes underlying the disease, intervention programs, patient focused education, and self management protocols. This journal is indexed on PubMed Central, MedLine and CAS. The manuscript management system is completely online and includes a very quick and fair peer-review system, which is all easy to use. Visit http://www. dovepress.com/testimonials.php to read real quotes from published authors.

Submit your manuscript here: https://www.dovepress.com/international-journal-of-chronic-obstructive-pulmonary-disease-journal 